\title{
The Interleukin 1 Receptor Type 1 Variant rs2234651 is a Risk Factor for Coronary Artery Disease in a Chinese Han Population: A Case Control Study
}

Jiayi Gu

Nanjing Medical University Second Affiliated Hospital

Ping Zhou

Nanjing Medical University Second Affiliated Hospital

Yongyue Wei

School of Public Health, Nanjing Medical University

Wei Gao

Sir Run Run Hospital, Nanjing Medical University

\section{Yujiao Yang}

Nanjing Medical University Second Affiliated Hospital

Ying Liu

Nanjing Medical University Second Affiliated Hospital

Lijuan Wang

Nanjing Medical University Second Affiliated Hospital

Xiang Lu

Sir Run Run Hospital, Nanjing Medical University

Zhengxia Liu ( $\nabla$ zhengxl1@njmu.edu.cn )

Nanjing Medical University Second Affiliated Hospital https://orcid.org/0000-0002-2132-6064

\section{Research article}

Keywords: coronary artery disease; IL-1R1; gene polymorphism; rs2234651

Posted Date: March 17th, 2020

DOI: https://doi.org/10.21203/rs.2.18857/v2

License: (1) This work is licensed under a Creative Commons Attribution 4.0 International License. Read Full License 


\section{Abstract}

Background While increased expression of soluble CD121a (Interleukin 1 Receptor Type 1, IL-1R1) has been shown to be significantly correlated with the severity of coronary artery disease (CAD), it is not yet clear whether IL-1R1 gene variants impact CAD pathogenesis. Methods The present study evaluated the effects of IL-1R1 variants on CAD in a Chinese-Han population; A two-stage case-control study assessed 928 Chinese-Han patients via coronary arteriography for coronary atherosclerosis. The allele and genotype frequencies of the analyzed IL-1R1 polymorphisms were determined via a polymerase chain reaction assay and directly sequenced. Results The IL-1R1 variant rs2234651 was found to be associated with both CAD risk and severity (the degree of exhibited vascular stenosis/number of affected vessels, $\mathrm{P}=$ 0.034; Gensini scoring, $P=0.006$ ). Furthermore, significant increase of IL-8 and IL-11 in patients carrying the CT+TT genotype was observed. Finally, serum CD121a levels significantly increased in patients with CAD that harbored the CT+TT genotype $(P<0.05)$. Conclusions These results demonstrate that the IL$1 \mathrm{R} 1$ variant rs2234651 is significantly associated with CAD risk and severity in the analyzed Chinese-Han population and rs2234651 may be a valuable target for CAD prevention and/or treatment.

\section{Background}

Coronary artery disease (CAD) is the leading cause of morbidity and mortality worldwide [1], the cost of caring for patients with cardiovascular disease has been shown to incur a significant impact on the global economy [2,3]; thus, novel and effective diagnostic and prognostic methods for CAD are urgently needed.

CAD is well established as a chronic inflammatory disease [4], and its pathogenesis has been shown to be driven by atherosclerosis, plaque rupture, and thrombosis [5-7]. Many inflammatory factor genes are now known to influence the development of atherosclerosis and coronary heart disease at genetic level [8-9]. Of these, IL-1 is a classical pro-inflammatory factor [10], and increased IL-1 level has been shown to result in a significantly increased CAD risk [11].

IL-1 consists of three ligands, IL-1 a, IL-1 $1 \beta$ and IL-1 receptor antagonist. IL-1R1 associates with the IL-1 receptor accessory protein (IL1RAP) and myeloid differentiation factor 88, forming a complex that transduces a signal and is responsible for most IL-1-mediated inflammatory responses [12-15]. The soluble CD121a is a truncated form of IL-1R1 and it has the identical binding affinity as the membrane form [16]. As such, it may act as a competitive inhibitor and does not initiate the IL-1 signal [17, 18].

Previous reports have indicated that $I L-1 R 1$ genetic polymorphisms are associated with many inflammatory diseases, such as atopic asthma, alcoholic liver disease, hematogenous osteomyelitis, invasive pneumococcal disease, and IgA nephropathy [19-23]. A lot of research also have shown that noncoding single nucleotide polymorphisms (SNPs) have functional effects [24-26], even more, Saykin et al. found that IL 1RAP rs12053868, not a coding SNP, is associated with decreased IL1RAP expression in the cortex and hippocampus, thereby increasing amyloidosis [14]. Thus, this case-control study explored whether the IL-1R1 non-coding region SNPs rs956730, rs2234650, rs2234651, and rs2287048 are 
associated with an increased risk of CAD in a Chinese population, and this study is the first to explore the relationship between $I L-1 R 1$ variations and CAD.

\section{Methods}

The present study enrolled 928 patients who underwent coronary arteriography (CAG) for coronary atherosclerosis at the Cardiology Department of the Second Affiliated Hospital of Nanjing Medical University between January 2013-December 2017. Of these, 478 patients enrolled between January 2013-December 2015 were assessed during the discovery phases of the study, while 450 subjects enrolled between January 2016-December 2017 were evaluated during the validation phase of the study.

The inclusion criteria were patients with suspected CAD who underwent coronary angiography, the exclusion criteria stipulated that patients have been previously diagnosed with congenital heart disease, cardiomyopathy, valvar heart disease, thrombotic disease, diffuse intravascular coagulation, liver disease, kidney failure, and/or have undergone a recent surgery or injury, and/or have a history of malignant tumors or other infectious diseases (such as sepsis or pneumonia)

All coronary angiograms were conducted by inserting a Judkins catheter into the right radial artery, and the produced results were independently reviewed by two experienced angiographers, both of whom were blinded to corresponding genotyping data. Patients were categorized according to their degree of coronary stenosis, such that they were determined to have 'no CAD', 'nonobstructive CAD', or 'significant CAD' if they exhibited $0 \%, 1-49 \%$, or $\geq 50 \%$ luminal narrowing in any epicardial coronary artery, respectively, as reported in the CSBC Registry [27]. In our study, 'CAD' is composed of 'nonobstructive CAD' and 'significant CAD'.

In the vessel scoring, stenosis $<50 \%$ in any major coronary artery was defined as 0 -vessel disease; having $\geq 50 \%$ stenosis in the luminal diameter of one or more coronary arteries was defined as a significant coronary lesion. Major coronary artery disease included left main coronary artery disease (considered a two-vessel disease characterized by stenosis $\geq 50 \%$ ), left anterior descending artery disease, left circumflex artery disease, and right coronary artery disease [28].

The severity and extent of coronary atherosclerosis was assessed via angiographic Gensini scoring [29], such that $0-24 \%, 25-49 \%, 50-74 \%, 75-89 \%$, and $90-99 \%$ angiographic stenosis was indicated by a score of $1,2,4,8$, and 16 points, respectively, while total occlusion was indicated by a score of 32 points. Each stenosed segment was then assessed and assigned a score between 0.5 and 5 , dependent upon the functional significance of the area supplied by that segment. These scores were multiplied by the coefficient defined for each coronary artery and segment, and the results then summed. Finally, the patients were divided into four groups, comprising those that scored a total of $0,0.5-19.5,20-39.5$, and $\geq 40$ points.

Patients with a systolic blood pressure $\geq 140 \mathrm{mmHg}$, and/or a diastolic blood pressure $\geq 90 \mathrm{mmHg}$, and/or who were currently using antihypertensive drugs were defined as being hypertensive. Those that 
exhibited a fasting plasma glucose level $\geq 7.0 \mathrm{mmol} / \mathrm{l}$, and/or that were currently using anti-diabetic drugs were defined as being diabetic. Finally, patients who exhibited high serum total cholesterol (TC) levels (TC $\geq 5.17 \mathrm{mmol} / \mathrm{l})$, and/or high triglyceride (TG) levels $(T G \geq 1.70 \mathrm{mmol} / \mathrm{l})$, and/or that were currently using lipid-lowering drugs were defined as having hyperlipemia.

The blood pressure, height, and body mass of each subject was measured upon admission. Prior to CAG assessment, blood samples were drawn into an inertia separation gel coagulation tube immediately after vascular puncture. TC, TG, high-density lipoprotein-cholesterol (HDL-C) and low-density lipoproteincholesterol (LDL-C) concentrations were determined by the Clinical Laboratory of the Second Affiliated Hospital of Nanjing Medical University, using routine clinical methods. Serum CD121a, IL-8, IL-11 and IL$1 \beta$ were measured using the CBA Human Soluble Protein Detection Kit (BD Biosciences, New Jersey, USA).

Genomic DNA was isolated from patient venous blood leukocyte pellets using a genomic DNA isolation kit (Applied Biomiga, San Diego, USA). Primers were designed using primer premier5 (Applied Premier, Vancouver, British Columbia, Canada) software to target rs2287048 (forward, 5'actaggcctcagtttcccactctata-3'; reverse, 5'-gtccaagactggaaatgtgtaatgg-3'), and rs956730, rs2234650, and rs2234651 (forward, 5'-caggttgcaagacagcttttagg-3'; reverse, 5'-cttatgcccaccagctcacttt-3'). The thermal cycling conditions were as follows: denaturation at $94^{\circ} \mathrm{C}$ for $3 \mathrm{~min}$; followed by 35 cycles of $94^{\circ} \mathrm{C}$ for $30 \mathrm{~s}$, $64^{\circ} \mathrm{C}$ for $30 \mathrm{~s}$, and $72^{\circ} \mathrm{C}$ for $1 \mathrm{~min}$; and a final extension at $72^{\circ} \mathrm{C}$ for $7 \mathrm{~min}$. Generated products were (570bp for rs2287048; 484bp for rs956730, rs2234650, and rs2234651) stored at $4^{\circ} \mathrm{C}$. Finally, the polymerase chain reaction products were identified via direct sequencing using an $A B I 3730 X L$ DNA Sequencer (Applied Biosystems, New York, USA).

All statistical analyses were performed using Statistical Package for the Social Sciences version 22.0(SPSS, Inc., Chicago, IL, USA) software. Continuous variables with normal distribution were expressed as the mean \pm standard deviation, and compared by unpaired Student's $t$-test. Continuous variables with abnormal distribution were analyzed by Mann-Whitney test. Categorical variables were presented as frequency ( $\mathrm{n}$ ) and proportion (\%) which were analyzed by Chi-square test.

Genotype distributions were tested at each polymorphic locus for deviation from Hardy-Weinberg equilibrium, and Haploview software (version 4.2, Broad Institute, Cambridge, MA) was used to assess linkage disequilibrium (LD). The chromatogram of direct sequencing was prepared using BioEdit 7.2.6.1 software. To minimize the effect of age and gender differences between patients with and without CAD, Propensity Score Matching (PSM) was performed for the population.

Association between genotypes and CAD severity (i.e. the degree of exhibited vascular stenosis, Gensini scoring and the number of affected vessels) was analyzed by Mann-Whitney or Kruskal-Wallis test.

Logistic regression model was used to estimate the association between genetic variants and the risk of CAD, and summarized as odds ratio (OR) and $95 \%$ confidence interval $(95 \% \mathrm{Cl})$, factors associated with the dependent variable at $\mathrm{P}<0.1$ on univariate analysis were then entered into the multivariate model. $\mathrm{P}$ $<0.05$ is considered to indicate statistical significance. 


\section{Results}

\section{Participant characteristics}

The present study assessed 928 patients via CAG to identify the location and extent of their arterial blockages (Figure 1). Of these, 343 patients with CAD, and 135 healthy controls (total, 478; enrolled January 2013-December 2015) were assessed during the discovery phases of the study. Compared to the healthy controls, the patients with CAD were older, and exhibited higher TG levels, and an increased prevalence of hypertension, diabetes mellitus, and hyperlipidemia (Table 1). After correcting for the effects of patient age variation, 135 of the patients with CAD displayed an estimated propensity score that matched (within the 0.05 caliper) with 135 of the healthy control patients. Of the 450 subjects assessed during the validation phase of the study (enrolled January 2016-December 2017), 159 were healthy controls, and 291 were determined to exhibit CAD.

\section{Genotype and allele frequencies}

The four analyzed $I L-1 R 1$ variants were used to evaluate the relationship between $I L-1 R 1$ genetic variants and risk of CAD; thus, their genotype distributions were assessed in both patients with and without CAD. The representative chromatogram of direct sequencing results were shown in Figure S1. Table S1 showed only SNP rs2234651 was initially found to be significantly associated with increased risk of CAD ( $P=$ 0.047), and furthermore, both the rs2234651 T allele and CT+TT genotype were found to be significantly associated with CAD after PSM correction, $(P=0.026$ and $P=0.025$, respectively). In contrast, no obvious differences were observed between the prevalence of the other three IL-1R1 SNPs in patients with compared to without CAD.

\section{Association between rs2234651 and CAD severity}

In this study, we further assessed whether the SNP rs2234651 was associated with an increased incidence of vascular lesions, and/or contributed to CAD severity (Table 2). Patients that harbored a variant (CT+TT) genome were shown an increasing severity of CAD (the degree of exhibited vascular stenosis/number of affected vessels, $P=0.034$; Gensini scoring, $P=0.006)$. Moreover, the frequency of the rs2234651 $\mathrm{T}$ allele was found to be increased among patients that displayed increasingly severe CAD (the degree of exhibited vascular stenosis, $P=0.024$; Gensini scoring, $P=0.005$; number of affected vessels, $P=0.021)$. These data indicate that patients that harbored a higher gene dosage of the rs2234651 T allele exhibited a significantly greater chance of developing coronary vessel lesions compared to healthy control patients, and also, indicate that the rs2234651 minor T allele was associated with a higher Gensini score, and an increased number of affected vessels.

We also analyzed the relationship of the rs2234651 SNP and inflammatory markers IL-8, IL-11, and IL-1 $\beta$. As shown in Figure $2 \mathrm{a}-\mathrm{C}$, we found a rising trend in serum IL-8, IL-11 and IL-1 $\beta$ in patients that carried the CT+TT genotype, especially for IL-8 and IL-11 ( $P=0.017$ and $P=0.014$, respectively).

\section{Haplotype analysis}


Next, we performed haplotype analysis. The LD analysis of rs956730, rs2234650, rs2234651, and rs22387048 was performed using the software Haploview 4.2, and the results are shown in Figure 3. Rs956730, rs2234650, and rs2234651 were located in the same LD block, and these 3 loci can be combined to produce 8 haplotypes. Haplotype was represented with the allele order of rs956730, rs2234650, and rs2234651. All alleles with frequency $<0.03$ were ignored in the analysis. As shown in Table S2, no haplotype was significantly associated with the risk of CAD.

\section{Validation phase of the study}

We further analyzed 450 patients undergone CAG between January 2016 and December 2017 at the Second Affiliated Hospital of Nanjing Medical University. Assessment of the patients in the validation phases revealed that compared with the healthy control subjects, the patients with CAD were older, more often male, and exhibited an increased prevalence of hypertension and diabetes mellitus and decreased TG and LDL-C levels (likely due to the use lipid-lowering drugs). After correcting for the effects of patient age and gender variation, 157 of the patients with CAD displayed an estimated propensity score that matched (within the 0.05 caliper) with 153 of the healthy control patients (Table S3).

CAD patients with rs2234651 SNP (CT+TT) were also shown to have a higher Gensini score, and more number of affected vessels were exhibited by the validation-phase patients $(P=0.016$ and $P=0.025$, respectively; Figure 4).

\section{Association between rs2234651 variants and serum CD121a levels}

The study further examined the relationship between serum CD121a expression and the rs2234651 SNP of CAD patients in the verification stage. As shown in Figure 2d, serum CD121a expression was increased in patients that carried the CT+TT genotype $(P=0.009)$.

\section{Discussion}

To the best of our knowledge, the present study is the first to examine the relationship between $I L-1 R 1$ variation and CAD. The generated results revealed a significant positive association between the IL-1RT SNP rs2234651 and the risk of CAD in the analyzed population, and this notably persisted after adjusting for various known CAD risk factors. Besides, patients that harbored the rs2234651 CT+TT genotype were found to exhibit increased serum CD121a levels. Furthermore, we also found a significant increase in serum IL-8 and IL-11 level in patients that carried the CT+TT genotype.

Since the rs2234651 variant is located within the IL-1R1 promoter region (NM_001320982.1 transcript, chromosome 2), and we have found patients that harbored the rs2234651 CT+TT genotype exhibited increased serum CD121a levels, we speculate that this variant may alter the ability of transcription factors to bind and up-regulate IL-1R1, and promoting IL-1//IL-1R1 inflammatory pathway. As well as, we observed a significant increase in serum IL-8 and IL-11 level in patients that carried the CT+TT genotype. These results are consistent with what we previously reported, serum IL-8 expression was increased in 
acute myocardial infarctions (AMI) patients, and a higher serum IL-11 level was found in unstable angina pectoris group [30]. Other research groups have demonstrated that high levels of circulating IL-8 were associated with large infarct size, impaired recovery of left ventricular function, and adverse clinical outcome in patients with CAD [31-35]; Yao et al. showed IL-11 was increased in thoracic aorta and plasma of acute thoracic aortic dissection (TAD) patients and may be a promising biomarker for diagnosis in patients with TAD [36]. These results suggest that the rs2234651 variant is associated with the proinflammatory state, thereby increasing the severity of CAD.

As shown in previous studies, IL-1R1 is a necessary component of the IL-1ß/IL1RAP pathway, and thus, increased IL-1R1 production is likely associated with IL-1 $\beta$-mediated inflammation and contribute to the development of CAD $[15,37,38]$. For example, Gehrke et al. demonstrated that inhibiting IL-1R1 with IL-1ra (anakinra) is sufficient to attenuate the severity of acute heart failure [39]. Loss of IL-1 signaling in IL-1R1

$(-/)$ mice results in a reduced rate of leukocyte infiltration, and thereby a reduced rate of myocardial infarctions [40], and decreased levels of atherosclerotic plaques [41]. Previous study by our research group has demonstrated that serum CD121a levels are elevated in patients with CAD, and particularly in those with $A M I$, such that increasing serum CD121a levels are associated with an increased CAD risk [30]. These results are consistent with our findings that patients harbored a variant (CT+TT) genome display a higher serum CD121a levels and increasing risk of CAD in this study. As a competitive inhibitor of the IL-1 proinflammatory pathway, we speculate that the elevated level of CD121a could be a protective compensation reaction in vivo.

Together, the results of the present study suggest that rs2234651 is likely a biologically functional variant associated with an increased CAD risk in the analyzed Chinese-Han population.

Some limitations of the present study have to be listed, such as the small sample size, single-center design, and retrospective nature. Although we have found that rs2234651 CT+TT genotypes are associated with elevated serum levels of CD121a, IL-8 and IL-11, we must consider that elevated levels of inflammatory factors may be a secondary factor in the severity of CAD rather than a direct result of risk genotypes. Thus, additional studies need to be conducted to confirm the association of rs2234651 with CAD severity.

\section{Conclusions}

These results suggest that the IL-1R1 variant rs2234651 is likely a CAD risk factor, and thus, rs2234651 will be a potential therapeutic target and/or diagnostic/prognostic biomarker for CAD and may be further investigated to develop improved methods for the prevention and treatment of this disease.

\section{Abbreviations}

IL-1R1: interleukin 1 receptor type 1

CAD: coronary artery disease 
SNP: single nucleotide polymorphism

IL1RAP: coronary arteriography

TC: total cholesterol

TG: triglyceride

HDL-C: high-density lipoprotein-cholesterol

LDL-C: low-density lipoprotein-cholesterol

LD: linkage disequilibrium

PSM: propensity score matching

OR: odds ratio

95\%Cl: 95\% confidence interval

AMl: acute myocardial infarctions

TAD: thoracic aortic dissection

\section{Declarations}

\section{Ethics Approval and Consent to Participate}

All subjects gave their informed consent for inclusion before they participated in the study (Additional file 1). The study was conducted in accordance with the Declaration of Helsinki, and the protocol was approved by the Ethics Committee of the Second Affiliated Hospital of Nanjing Medical University, Jiangsu, China (2012-KY-027, Additional file 2).

\section{Consent to publish}

Not applicable.

\section{Availability of Data and Materials}

The datasets used and/or analysed during the current study are available from the corresponding author on reasonable request.

\section{Competing Interests}

The authors declare that they have no competing interests.

\section{Funding}


This work was supported by grants from the National Natural Sciences Foundation of China ( No. 81770440 , No. 81470501, No. 81970374).

\section{Author Contributions}

$\mathrm{ZL}, \mathrm{XL}$ conceived and designed the experiments; JG, $\mathrm{YL}, \mathrm{PZ}, \mathrm{YY}$ and $\mathrm{LW}$ performed the experiments; $\mathrm{ZL}$, JG, YW analyzed the data; ZL, WG and XL contributed reagents/materials/analysis tools; JG, XL, ZL wrote the paper. All authors read and approved the final manuscript.

\section{Acknowledgments}

We would like to thank all study participants and their families, and to gratefully acknowledge the assistance of Dr. Lie Sun (sample collection), and Dr. Wenwen Guo (valuable advice regarding patient genetics).

\section{References}

1. Duan L, Liu C, Hu J, Liu Y, Wang J, Chen G, Li Z, Chen H: Epigenetic mechanisms in coronary artery disease: The current state and prospects. Trends Cardiovasc. 2018; 28: 311-319.

2. Mastoi QU, Wah TY, Gopal Raj R, Iqbal U: Automated Diagnosis of Coronary Artery Disease: A Review and Workflow. Cardiol Res Pract. 2018; doi: 10.1155/2018/2016282.

3. Duan L, Liu C, Hu J, Liu Y, Wang J, Chen G, Li Z, Chen H: Defining and Measuring a Standard Set of Patient-Relevant Outcomes in Coronary Artery Disease. Am. J. Cardiol. 2018; 121: 1477-1488.

4. Mury P, Faes C, Millon A, Mura M, Renoux C, Skinner S, Nicaise V, Joly P, Della Schiava N, Lermusiaux P, et al: Higher Daily Physical Activity Level Is Associated with Lower RBC Aggregation in Carotid Artery Disease Patients at High Risk of Stroke. Front Physiol. 2017; doi: 10.3389/fphys.2017.01043.

5. Rothenbacher D, Kleiner A, Koenig W, Primatesta P, Breitling LP, Brenner H: Relationship between inflammatory cytokines and uric acid levels with adverse cardiovascular outcomes in patients with stable coronary heart disease. PLoS ONE. 2012; 7: e45907.

6. Inoue T, Komoda H, Nonaka M, Kameda M, Uchida T, Node K: Interleukin-8 as an independent predictor of long-term clinical outcome in patients with coronary artery disease. INT J CARDIOL. 2008; 124: 319-325.

7. McCarty S, Frishman W: Interleukin 1beta: a proinflammatory target for preventing atherosclerotic heart disease. CARDIOL REV. 2014; 22: 176-181.

8. Zhang X, Pei F, Zhang M, Yan C, Huang M, Wang T, Han Y: Interleukin-17A gene variants and risk of coronary artery disease: a large angiography-based study. Clin. Chim. Acta. 2011;412: 327-331.

9. Kumari R, Kumar S, Ahmad MK, Singh R, Kant Kumar S, Pradhan A, Chandra S, Kumar S. Promoter variants of TNF-a rs1800629 and IL-10 rs1800871 are independently associated with the susceptibility of coronary artery disease in north Indian. Cytokine. 2018; 110: 131-136.

10. Dinarello CA:Biologic basis for interleukin-1 in disease. Blood. 1996; 87: 2095-2147. 
11. Naka KK, Bechlioullis A, Marini A, Sionis D, Vakalis K, Triantis G, Wilkins L, Rogus J, Kornman KS, Witztum JL, et al: Interleukin-1 genotypes modulate the long-term effect of lipoprotein(a) on cardiovascular events: The loannina Study. J Clin Lipidol. 2018; 12:338-347.

12. Wang D, Zhang S, Li L, Liu X, Mei K, Wang X: Structural insights into the assembly and activation of IL-1 $\beta$ with its receptors. NAT IMMUNOL. 2010; 11: 905-911.

13. M B, NG F: The role of IL-1 in the pathogenesis of heart disease. ARCH IMMUNOL THER EX. 2009; 57: 165-176.

14. Ramanan VK, Risacher SL, Nho K, Kim S, Shen L, McDonald BC, Yoder KK, Hutchins GD, West JD, Tallman EF, et al: GWAS of longitudinal amyloid accumulation on 18F-florbetapir PET in Alzheimer's disease implicates microglial activation gene IL1RAP. Brain. 2015; 138: 3076-3088.

15. Beaulieu LM, Lin E ,Mick E, Koupenova M, Weinberg EO, Kramer CD, Genco CA, Tanriverdi K, Larson MG, Benjamin EJ, et al:Interleukin 1 receptor 1 and interleukin 1 beta regulate megakaryocyte maturation, platelet activation, and transcript profile during inflammation in mice and humans. Arterioscler. Thromb. Vasc. Biol. 2014; 34: 552-564.

16. Dower SK, Wignall JM, Schooley K, McMahan CJ, Jackson JL, Prickett KS, Lupton S, Cosman D, Sims JE: Retention of ligand binding activity by the extracellular domain of the IL-1 receptor. J. Immunol. 1989; 142: 4314-4320.

17. Bernstein SH, Fay J, Frankel S, Christiansen N, Baer MR, Jacobs C, Blosch C, Hanna R, Herzig G: A phase I study of recombinant human soluble interleukin-1 receptor (rhu IL-1R) in patients with relapsed and refractory acute myeloid leukemia. Cancer Chemother. Pharmacol. 1999; 43: 141-144.

18. Sims JE, Giri JG, Dower SK: The two interleukin-1 receptors play different roles in IL-1 actions. Clin. Immunol. Immunopathol. 1994; 72: 9-14.

19. Chen J, Zhang J, Hu H, Jin Y, Xue M: Polymorphisms of RAD50, IL33 and IL1RL1 are associated with atopic asthma in Chinese population. Tissue Antigens. 2015; 86: 443-447.

20. Novo-Veleiro I, Cieza-Borrella C, Pastor I, González-Sarmiento R, Laso FJ, Marcos M: Analysis of the relationship between interleukin polymorphisms within miRNA-binding regions and alcoholic liver disease. Rev Clin Esp. 2018; 218: 170-176.

21. Osman AE, Mubasher M, EISheikh NE, AlHarthi H, AlZahrani MS, Ahmed N, ElGhazali G, Bradley BA, Fadil AS: Association of single nucleotide polymorphisms in pro-inflammatory cytokine and toll-like receptor genes with pediatric hematogenous osteomyelitis. Genet. Mol. Res. 2016; doi: 10.4238/gmr.15027718.

22. Sangil A, Arranz MJ, Güerri-Fernández R, Pérez M, Monzón H, Payeras A, Andrés M, Torviso J, Ibañez L, Garau J, et al: Genetic susceptibility to invasive pneumococcal disease. Infect. Genet. Evol. 2018; 59: 126-131.

23. Xie M, Zhang D, Zhang Y, Yang X, Su Y, Wang Y, Huang H, Han H, Li W, Fu K, et al: Association of genetic polymorphisms in IL-1R1 and IL-1R2 genes with IgA nephropathy in the Han Chinese population. Oncotarget. 2017; 8: 50673-50679. 
24. Kapranov P, Willingham AT, Gingeras TR: Genome-wide transcription and the implications for genomic organization. Nat. Rev. Genet. 2007; 8: 413-423.

25. An integrated encyclopedia of DNA elements in the human genome. Nature. 2012; 489: 57-74.

26. De Jager PL, Srivastava G, Lunnon K, Burgess J, Schalkwyk LC, Yu L, Eaton ML, Keenan BT, Ernst J, McCabe C, et al: Alzheimer's disease: early alterations in brain DNA methylation at ANK1, BIN1, RHBDF2 and other loci. Nat. Neurosci. 2014; 17: 1156-1163.

27. Samman Tahhan A, Sandesara P, Hayek SS, Hammadah M, Alkhoder A, Kelli HM, Topel M, O'Neal WT, Ghasemzadeh N, Ko YA, et al: High-Sensitivity Troponin I Levels and Coronary Artery Disease Severity, Progression, and Long-Term Outcomes. J Am Heart Assoc. 2018; doi: 10.1161/JAHA.117.007914.

28. Wang L, Chen J, Zeng Y, Wei J, Jing J, Li G, Su L, Tang X, Wu T, Zhou L. Functional Variant in the SLC22A3-LPAL2-LPA Gene Cluster Contributes to the Severity of Coronary Artery Disease. Arterioscler. Thromb. Vasc. Biol. 2016; 36: 1989-1996.

29. Gensini GG: A more meaningful scoring system for determining the severity of coronary heart disease. Am. J. Cardiol. 1983; 51: 606.

30. Liu Z, Zhang M, Wu J, Zhou P, Liu Y, Wu Y, Yang Y, Lu X: Serum CD121a (Interleukin 1 Receptor, Type I): A Potential Novel Inflammatory Marker for Coronary Heart Disease. PLoS ONE. 2015; 10: e0131086.

31. van der Pouw Kraan TC, Bernink FJ, Yildirim C, Koolwijk P, Baggen JM, Timmers L, Beek AM, Diamant M, Chen WJ, van Rossum AC: Systemic toll-like receptor and interleukin-18 pathway activation in patients with acute ST elevation myocardial infarction. J. Mol. Cell. Cardiol. 2014; 67: 94-102.

32. Shetelig, C., Limalanathan, S., Hoffmann, P., Seljeflot, I., Gran, J. M., Eritsland, J., Andersen, G. $\varnothing$. Association of IL-8 With Infarct Size and Clinical Outcomes in Patients With STEMI. J. Am. Coll. Cardiol. 2018; 72: 187-198.

33. Shetelig C, Limalanathan S, Hoffmann P, Seljeflot I, Gran JM, Eritsland J, Andersen GØ: Inflammatory cytokines in chronic heart failure: interleukin-8 is associated with adverse outcome. Results from CORONA. Eur. J. Heart Fail. 2014; 16: 68-75.

34. Cavusoglu E, Marmur JD, Yanamadala S, Chopra V, Hegde S, Nazli A, Singh KP, Zhang M, Eng C: Elevated baseline plasma IL-8 levels are an independent predictor of long-term all-cause mortality in patients with acute coronary syndrome. Atherosclerosis. 2015; 242: 589-594.

35. noue T, Komoda H, Nonaka M, Kameda M, Uchida T, Node K: Interleukin-8 as an independent predictor of long-term clinical outcome in patients with coronary artery disease. Int. J. Cardiol. 2008; 124: 319325.

36. Xu Y, Ye J, Wang M, Wang Y, Ji Q, Huang Y, Zeng T, Wang Z, Ye D, Jiang H, et al: Increased interleukin11 levels in thoracic aorta and plasma from patients with acute thoracic aortic dissection. Clin. Chim. Acta. 2018; 481: 193-199.

37. Bhaskar V, Yin J, Mirza AM, Phan D, Vanegas S, Issafras H, Michelson K, Hunter JJ, Kantak SS: Monoclonal antibodies targeting IL-1 beta reduce biomarkers of atherosclerosis in vitro and inhibit 
atherosclerotic plaque formation in Apolipoprotein E-deficient mice. Atherosclerosis. 2011; 216: 313320.

38. Chi H, Messas E, Levine RA, Graves DT, Amar S: Interleukin-1 receptor signaling mediates atherosclerosis associated with bacterial exposure and/or a high-fat diet in a murine apolipoprotein $\mathrm{E}$ heterozygote model: pharmacotherapeutic implications. Circulation. 2004; 110: 1678-1685.

39. Gehrke N, Hövelmeyer N, Waisman A, Straub BK, Weinmann-Menke J, Wörns MA, Galle PR, Schattenberg JM: Hepatocyte-specific deletion of IL1-RI attenuates liver injury by blocking IL-1 driven autoinflammation. J. Hepatol. 2018; 68: 986-995.

40. Saxena A, Chen W, Su Y, Rai V, Uche OU, Li N, Frangogiannis NG: IL-1 induces proinflammatory leukocyte infiltration and regulates fibroblast phenotype in the infarcted myocardium. J. Immunol. 2013; 191: 4838-4848.

41. Chamberlain J, Francis S, Brookes Z, Shaw G, Graham D, Alp NJ, Dower S, Crossman DC: Interleukin-1 regulates multiple atherogenic mechanisms in response to fat feeding. PLoS ONE. 2009; 4: e5073.

\section{Tables}

Table 1. Baseline characteristics of discovery-phase patients 


\begin{tabular}{|c|c|c|c|c|c|c|}
\hline & \multicolumn{2}{|c|}{ Before PSM } & \multirow[t]{2}{*}{$P$} & \multicolumn{2}{|c|}{ After PSM } & \multirow[t]{2}{*}{$P$} \\
\hline & $\begin{array}{l}\text { No CAD } \\
(n=135)\end{array}$ & $\begin{array}{c}\text { CAD } \\
(n=343)\end{array}$ & & $\begin{array}{l}\text { No CAD } \\
(n=135)\end{array}$ & $\begin{array}{c}\text { CAD } \\
(n=135)\end{array}$ & \\
\hline Age (yr) & $58.72 \pm 9.66$ & $64.30 \pm 9.70$ & $0.000^{* * *}$ & $58.72 \pm 9.66$ & $59.47 \pm 9.16$ & 0.541 \\
\hline $\begin{array}{c}\text { Gender } \\
\text { (male n (\%)) }\end{array}$ & $\begin{array}{c}71 \\
(52.59)\end{array}$ & $\begin{array}{c}183 \\
(53.35)\end{array}$ & 0.881 & $\begin{array}{c}71 \\
(52.60)\end{array}$ & $\begin{array}{c}81 \\
(60.00)\end{array}$ & 0.22 \\
\hline \multicolumn{7}{|l|}{ Laboratory data } \\
\hline $\begin{array}{c}\mathrm{TC} \\
(\mathrm{mmol} / \mathrm{L})\end{array}$ & $4.48 \pm 1.10$ & $4.64 \pm 1.15$ & 0.165 & $4.48 \pm 1.10$ & $4.66 \pm 1.18$ & 0.71 \\
\hline $\begin{array}{c}\text { TG } \\
(\mathrm{mmol} / \mathrm{L})\end{array}$ & $1.45 \pm 0.80$ & $1.64 \pm 0.96$ & $0.042^{*}$ & $1.45 \pm 0.80$ & $1.71 \pm 1.06$ & 0.079 \\
\hline $\begin{array}{c}\text { HDL-C } \\
(\mathrm{mmol} / \mathrm{L})\end{array}$ & $1.18 \pm 0.29$ & $1.18 \pm 0.30$ & 0.948 & $1.18 \pm 0.29$ & $1.17 \pm 0.28$ & 0.833 \\
\hline $\begin{array}{c}\text { LDL-C } \\
(\mathrm{mmol} / \mathrm{L})\end{array}$ & $2.55 \pm 0.80$ & $2.65 \pm 0.85$ & 0.246 & $2.55 \pm 0.80$ & $2.67 \pm 0.88$ & 0.324 \\
\hline Coronary risk fa & actors n (\%) & & & & & \\
\hline Hypertension & $\begin{array}{c}77 \\
(57.04)\end{array}$ & $\begin{array}{c}241 \\
(70.26)\end{array}$ & $0.006^{* *}$ & $\begin{array}{c}77 \\
(57.00)\end{array}$ & $\begin{array}{c}89 \\
(65.90)\end{array}$ & 0.133 \\
\hline Diabetes & 18 & 103 & $0.000^{* * *}$ & 18 & 49 & $0.000 * * *$ \\
\hline & $(13.30)$ & $(30.00)$ & & $(13.30)$ & $(36.30)$ & \\
\hline Hyperlipidemia & $\begin{array}{c}52 \\
(38.81)\end{array}$ & $\begin{array}{c}182 \\
(53.06)\end{array}$ & $0.004^{* *}$ & $\begin{array}{c}52 \\
(38.50)\end{array}$ & $\begin{array}{c}72 \\
(53.30)\end{array}$ & $0.015^{*}$ \\
\hline
\end{tabular}


Values are presented as the mean \pm standard deviation or $\mathrm{n}(\%)$.

CAD, coronary artery disease; HDL-C, high-density lipoprotein cholesterol; LDL-C, lowdensity lipoprotein cholesterol; PSM, Propensity Score Matching; TC, total cholesterol; TG, triglyceride; yr, years.

$* P<0.05$, ** $P<0.01$, and $* * * P<0.001$ indicate statistical significance.

Table 2. Association of rs2234651 with CAD 
Degree of exhibited vascular stenosis

N NO CAD $\quad$ Nonobstructive $\quad$ Significant CAD $P$ CAD

\begin{tabular}{cccccc}
\hline $\begin{array}{c}\text { Genotype } \\
\text { CC }\end{array}$ & 378 & 114 & 69 & 195 & $0.034^{*}$ \\
$\mathrm{n}(\%)$ & & $(84.4)$ & $(81.2)$ & $(75.6)$ & \\
\hline CT+TT & 100 & 21 & 16 & 63 & \\
$\mathrm{n}(\%)$ & & $(15.6)$ & $(18.8)$ & $(24.4)$ & \\
Allele & & & & & $0.024^{*}$ \\
$\mathrm{C}$ & 851 & 249 & 153 & 449 & \\
$\mathrm{n}(\%)$ & & $(92.2)$ & $(90.0)$ & $(87.0)$ & \\
$\mathrm{T}$ & 105 & 21 & 17 & 67 & \\
$\mathrm{n}(\%)$ & & $(7.8)$ & $(10.0)$ & $(13.0)$ & \\
\hline
\end{tabular}

Gensini Score

\begin{tabular}{ccccccc}
\hline & $\mathrm{N}$ & $\mathbf{0}$ & $\mathbf{0 . 5}-19.5$ & $20-39.5$ & $\geq 40$ & $P$ \\
\hline $\begin{array}{c}\text { Genotype } \\
\text { CC }\end{array}$ & 378 & 114 & 168 & 48 & 48 & $0.006^{* *}$ \\
n (\%) & & $(84.4)$ & $(80.8)$ & $(72.7)$ & $(69.6)$ & \\
\hline CT+TT & 100 & 21 & 40 & 18 & 21 & \\
n (\%) & & $(15.6)$ & $(19.2)$ & $(27.3)$ & $(30.4)$ & \\
Allele & & & & & & $0.005^{*}$ \\
C & 851 & 249 & 373 & 113 & 116 & \\
n (\%) & & $(92.2)$ & $(89.7)$ & $(85.6)$ & $(84.1)$ & \\
T & 105 & 21 & 43 & 19 & 22 & \\
n (\%) & & $(7.8)$ & $(10.3)$ & $(14.4)$ & $(15.9)$ & \\
\hline
\end{tabular}

Number of affected vessels

$\begin{array}{llllll}\mathbf{N} & 0 & 1 & 2 & 3 & P\end{array}$

\section{Genotype}

$\begin{array}{lllllll}\text { CC } & 378 & 183 & 108 & 45 & 42 & 0.034 *\end{array}$



n $(\%)$
(83.2)
$(77.1)$
(73.8)
(73.7)

$\begin{array}{cccccc}\mathrm{CT}+\mathrm{TT} & 100 & 37 & 32 & 16 & 15 \\ \mathrm{n}(\%) & & (16.8) & (22.9) & (26.2) & (26.3)\end{array}$

Allele

$\begin{array}{ccccccc}\mathrm{C} & 851 & 402 & 247 & 104 & 98 & 0.021^{*} \\ \mathrm{n}(\%) & & (91.4) & (88.2) & (85.2) & (86.0) & \\ \mathrm{T} & 105 & 38 & 33 & 18 & 16 & \\ \mathrm{n}(\%) & & (8.6) & (11.8) & (14.8) & (14.0) & \end{array}$

CAD, coronary artery disease. $* P<0.05$, and ${ }^{* *} P<0.01$ indicate statistical significance.

\section{Figures}

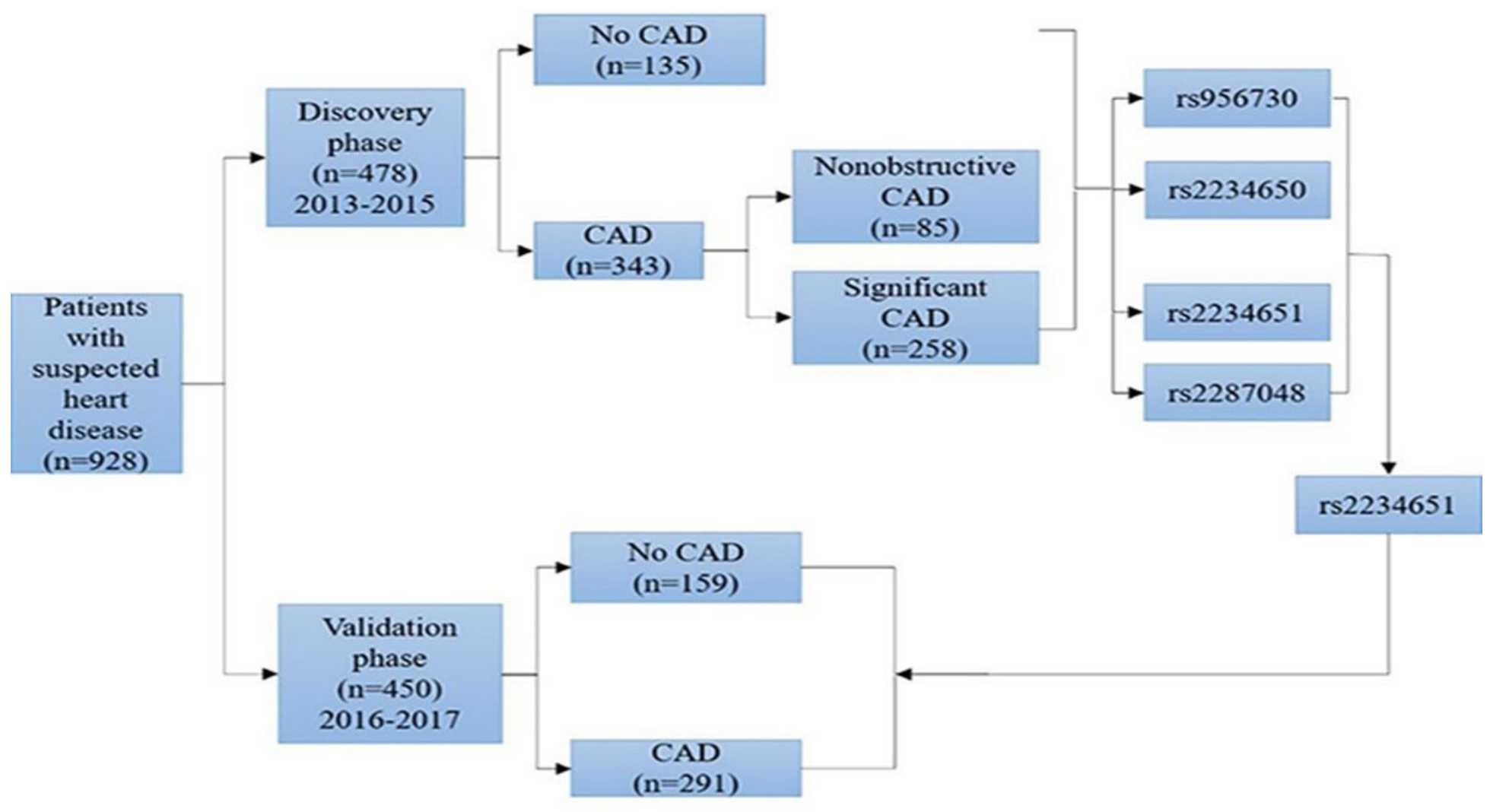

Figure 1

Study flow chart. The present study enrolled 928 patients with suspected heart disease and comprised both, the discovery and validation phases. CAD, coronary artery disease. 

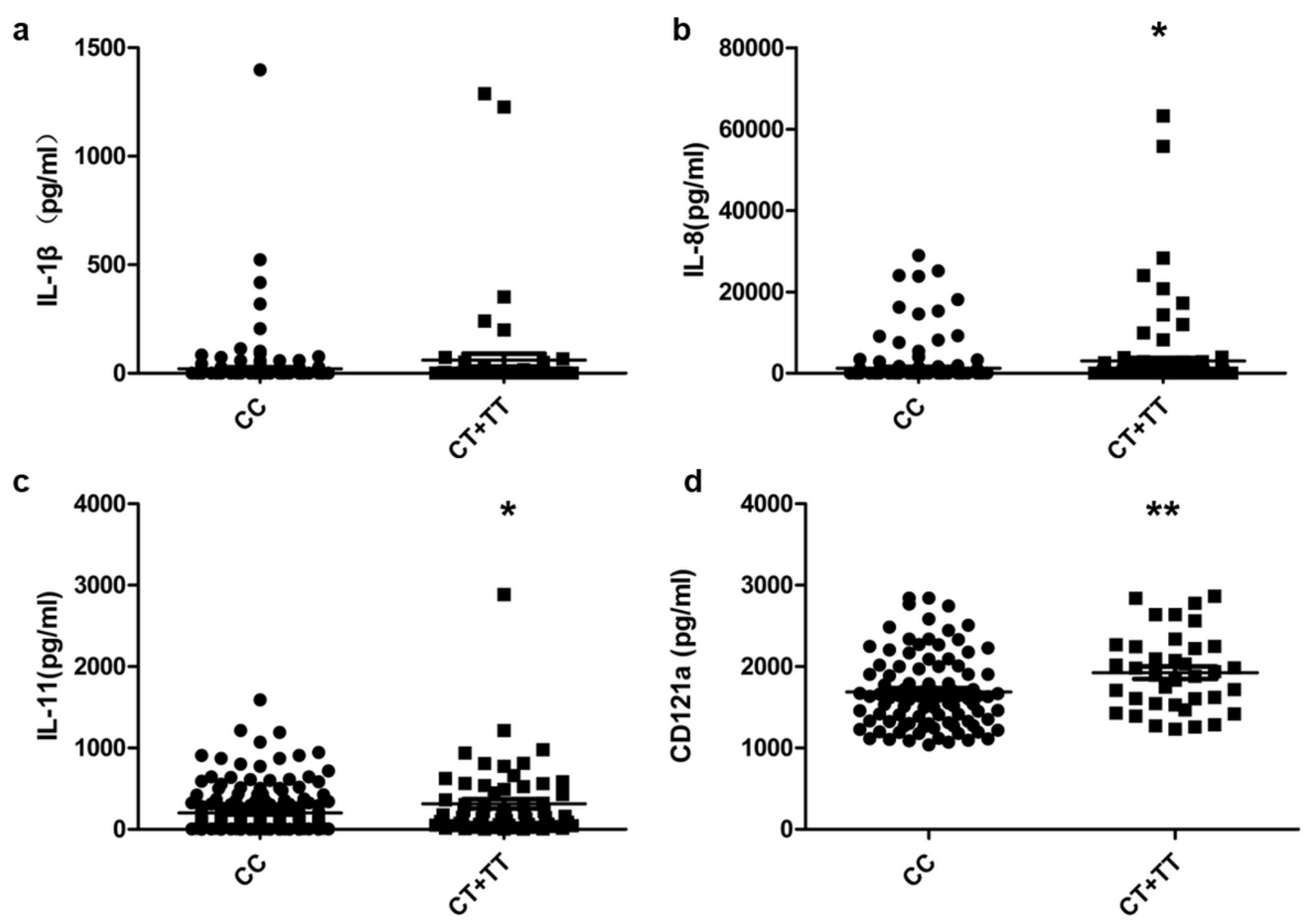

Figure 2

Relationship of the rs 2234651 SNP and inflammatory markers. (a) Serum IL-1 $\beta$ expression in patients that harbored the CC compared to the CT+TT genotype. (b) Serum IL-8 expression in patients that harbored the CC compared to the CT+TT genotype. (c) Serum IL-11 expression in patients that harbored the CC compared to the CT+TT genotype. (d) Serum CD121a expression in patients that harbored the CC compared to the CT+TT genotype. * $\mathrm{P}<0.05$, and ${ }^{\star *} \mathrm{P}<0.01$ indicate statistical significance. 
a

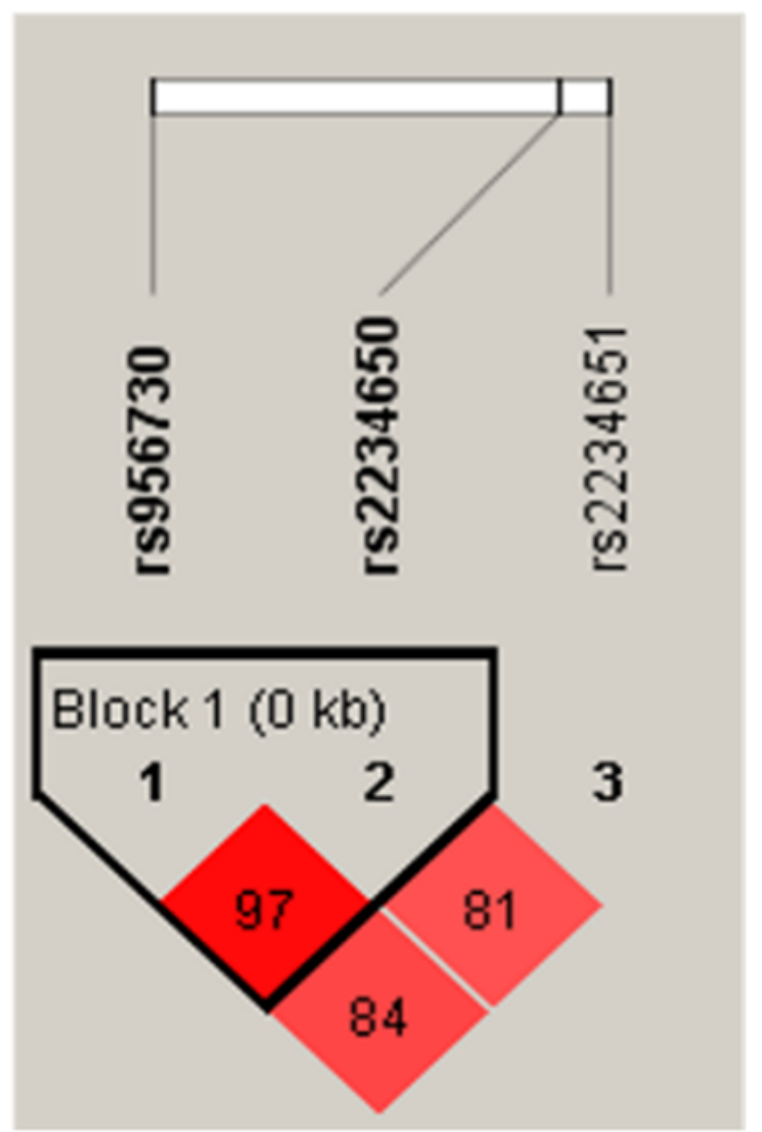

b

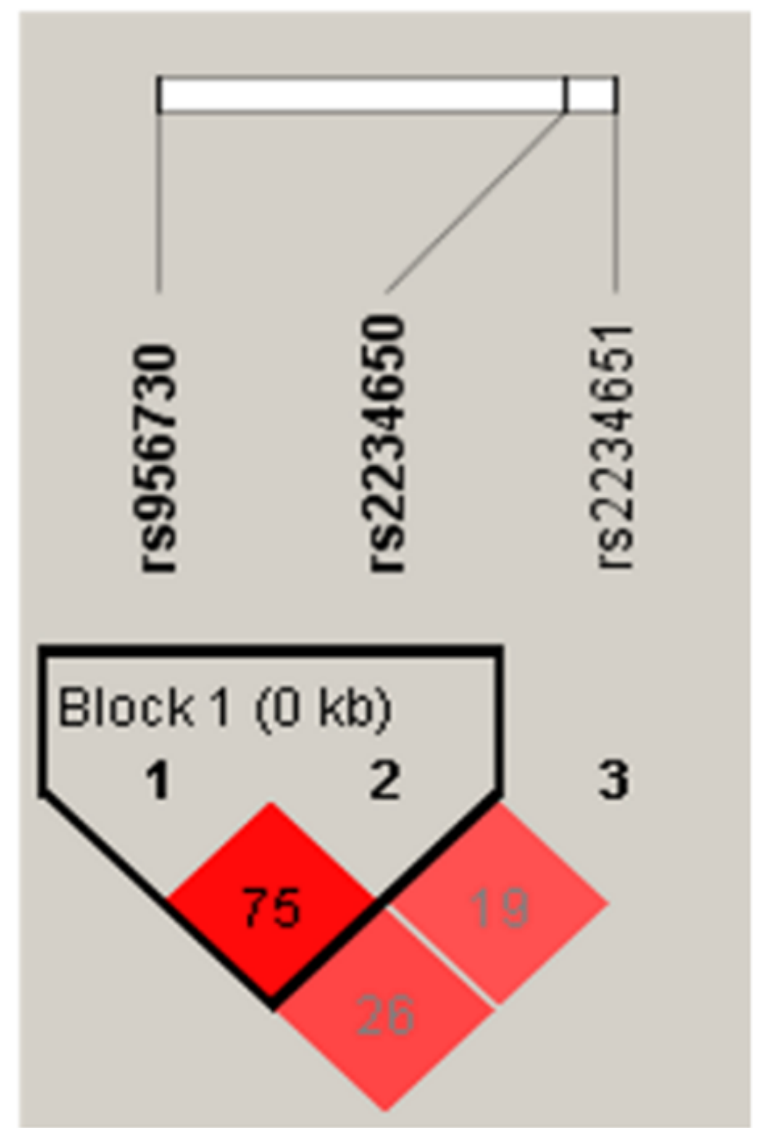

Figure 3

Linkage disequilibrium map of 3 SNPs ( rs956730, rs2234650 and rs2234651) in CD121a. (a): The boxes show the D' value; (b): The boxes show the r2 value.

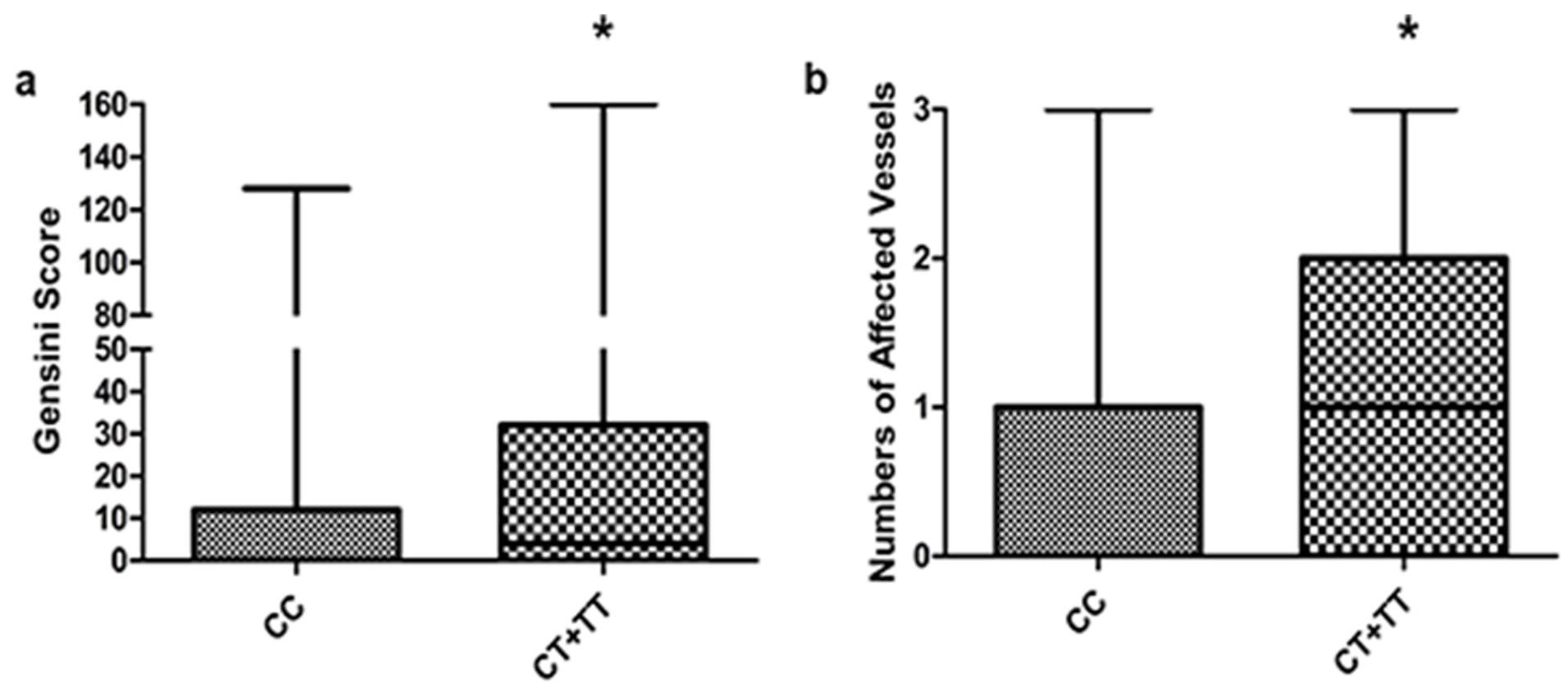


Figure 4

Assessment of rs2234651 distribution and CAD severity in the validation phases. (a) Gensini scores achieved by patients that harbored the CC compared to the CT+TT genotype. (b) The number of affected vessels observed in patients that harbored the CC compared to the CT+TT genotype. ${ }^{*} P<0.05$ indicates statistical significance.

\section{Supplementary Files}

This is a list of supplementary files associated with this preprint. Click to download.

- Tables2.docx

- Figures1.docx

- TableS3.docx

- TableS1.docx

- Additionalfile2.jpg

- FigureS2.docx

- Additionalfile1.docx

- Additionalfile3.docx 